\title{
Adjunct Use of Low Dose Risperidone Verses Adjunct Use of Clonazepam with an SSRI in Treatment of Moderate to Severe Anxiety States; A Comparative Study
}

Naurose Ali ${ }^{1, *}$, Aysha Rasheed ${ }^{1}$, Ismail Tariq ${ }^{2}$, Farukh Abdullah $^{1}$, Ayesha Sarwar ${ }^{1}$, Rizwan Atta ${ }^{1}$, Zahra Qammer ${ }^{1}$

${ }^{1}$ Department of Psychiatry, Lahore General Hospital, Lahore, Pakistan.

${ }^{2}$ Department of Psychiatry, Continental Medical College, Lahore, Pakistan.

\begin{abstract}
Background: The unprecedented and unaccounted use of benzodiazepines is running havoc in many countries in terms of substance abuse problems and is exponentially increasing. The prescription of benzodiazepines should be minimized and the search for and use of better alternatives be incorporated into clinical practice of Psychiatry.
\end{abstract}

Aim: The aim of this study is to compare the efficacy of low dose risperidone and relatively higher dosage of clonazepam in moderate to severe anxiety states when used in combination with an SSRI.

Method: A group of 60 patients having moderate to severe anxiety level, as measured by Hamilton Anxiety Rating Scale (HARS) were randomly divided into two groups. First group was given $1 \mathrm{mg}$ risperidone with $50 \mathrm{mg}$ Sertraline and the second group was given $3 \mathrm{mg}$ of clonazepam with $50 \mathrm{mg}$ Sertraline. The anxiety levels of patients in both groups were again calculated on the $10^{\text {th }}$ post treatment day and compared to each other. Moreover, the Side Effect profiling of Risperidone was also done in all the Patients by using the Glasgow Anti-psychotic Side-effect Scale (GASS)'.

Result: The first group had a decrease in the HARS scores having a value of 19.56 between day 0 and day 10 . The second group also showed a decrease in the anxiety levels with a mean HARS score of 31.12 on day 0 to 12.36 on day 10 . The mean value of decrease in HARS score was comparable.

Conclusion: The efficacy of low dose risperidone is comparable to that of relatively high dose of clonazepam in treating moderate to severe anxiety states. Use of risperidone for symptomatic relief from anxiety is a viable alternative to benzodiazepines.

\section{INTRODUCTION}

Anxiety is a state of apprehension and fear that can be felt both episodically or incessantly, depending on the nature of disorder. The cause of anxiety can be both psychological and physiological, therefore, it is important to make this distinction for the sake of management. Anxiety arises from the over-stimulation of sympathetic nervous system which also accounts for the wide range of somatic manifestations of anxiety states, such as palpitation, tachycardia, excessive sweating, fainting, nausea, feeling of urinary urgency, muscular tension etc. As a consequence of these physical complaints patients also experience a wide array of psychological complaints such as fear, apprehension, feeling of choking, heart attack and impending doom [1,2]. Patients with anxiety frequently find the performance of daily activities and tasks much more cumbersome and tedious than they normally would and also experience or perceive mild stresses in an exaggerated way [2].

*Address correspondence to this author at the Department of Psychiatry, Lahore General Hospital, Lahore, Pakistan.

E-mail: naurose_ali@yahoo.com
Anxiety symptoms can be associated with many psychiatric disorders. Mild, moderate and even severe anxiety states can be associated with as many as 90 percent patient with a primary DSM 5 diagnosis of Major Depressive Disorder [1]. Anxiety symptoms are also imperative with a primary diagnosis of Obsessive Compulsive Disorder along with depression and depersonalization, which are also commonly associated [3]. The content of obsessions in OCD, let alone, generates a lot of fear, guilt and anxiety, at times, the resistance of a compulsive act might increase the level of anxiety in the patient and performance of the compulsive act may also increase the level of anxiety [1]. Patients with Generalized Anxiety Disorder experience anxiety on a relentless basis [3].

Psychiatrist, all over the world, use various method in treating the co-morbid anxiety symptoms associated with the primary DSM diagnosis of Major Depressive Disorder, Generalized Anxiety Disorders and Obsessive Compulsive Disorder. Some common strategies include the use of Benzodiazepines, buspirone and various classes of anti-depressants [4]. 
Clonazepam is a widely used benzodiazepines for anxiolysis and has been selected for use in this study. It binds to GABAA receptors and increases the effect of the neurotransmitter GABA. It begins having an effect within an hour and lasts between six and 12 hours. Common side effects include sleepiness, poor coordination, and agitation. Long-term use may result in tolerance, dependence, and withdrawal symptoms if stopped abruptly.

Recently, the use of anti-psychotics in treatment of anxiety disorders has also exhibited an upward trend, mostly as augmentation therapy with drugs from other classes [5].

Risperidone, a second generation anti-psychotic, has been chosen for treatment of moderate to severe anxiety states in this clinical trial. Risperidone is widely used anti-psychotic and is used in the treatment of various psychotic disorders [6]. Risperidone is administered both in the form of oral tablets or in solution form. It is a D2 antagonist possessing anti-serotonergic, anti-adrenergic and anti-histaminergic properties. Adverse effects of risperidone include significant weight gain and metabolic problems such as diabetes mellitus type 2 as well as Extra-Pyramidal Symptoms (EPS) and neuroleptic malignant syndrome [6].

Risperidone acts as antagonist at D1 and D2 family [7]. This antagonism may account for its anti-psychotic actions as well as the various extra-pyramidal signs and symptoms that it causes [7]. Its action on serotonergic receptors especially 5HT2a and 5HT3c as antagonist may also account for its propensity to cause lower EPS than other neuroleptics and increase in metabolic syndrome and resultant weight gain7. Risperidone also acts on a variety of other receptors like Alpha $\alpha 1$ adrenergic receptors, Alpha $\alpha 2$ adrenergic receptors and Histamine $\mathrm{H} 1$ receptor [7]. Effects on these receptors may account for its sedation and reduction in alertness and agitation thereby reducing anxiety levels.

There has been a significant increase in the use of second generation anti-psychotics in anxiety disorder in the U.K over a period spanning from 1996 t0 2007 [5]. Using an extensive database of 4,166 office visits from 1996 to 2007, Comer et al. found that the proportion of visits for anxiety disorders in which an antipsychotic was prescribed increased from $10.6 \%$ to $21.3 \%$ over the 12 -year observation period [5]. This trend was paralleled by the introduction of several second-generation antipsychotics and the widespread adoption of this class of agents for psychotic and mood disorders [5]. The second-generation antipsychotics have fewer or less severe motor side effects than first-generation agents, which may account for their increased use for anxiety disorders despite the high rate of weight gain and metabolic side effects associated with some of the second-generation agents and the risk of cerebrovascular events in the elderly.

Moreover the use of anti-psychotics in anxiety disorders has also been suggested in various psychopharmacological algorithms and guidelines.

\section{AIM}

The aim of the study was to determine the efficacy of the usage of low dose Risperidone as compared to relatively higher dosage of Clonazepam in treating the associated anxiety symptoms in patients with DSM 5 established primary diagnosis of Major Depressive Disorder, Obsessive Compulsive Disorder and Generalized Anxiety Disorder.

The null-hypothesis devised for this study is:

"Risperidone use, in low dose, is not as effective in treating moderate to severe anxiety states associated with MDD, OCD and GAD as is Clonazepam in high dosage when used in combination with an SSRI".

\section{METHOD}

The sample for this study was taken from outdoor department of Punjab Institute of Mental Health, Lahore. A group of 60 patients was selected. There was no binding of sex, age and ethnicity. 24 patients had a primary DSM 5 diagnosis of Generalized Anxiety Disorder; 24 patients had a primary DSM 5 diagnosis of Major Depressive Disorder and the rest of the 12 patients had a primary DSM 5 diagnosis of Obsessive Compulsive Disorder. There was no binding on the number past episodes/relapses of the primary diagnosis that were assigned to the patients. Hamilton Anxiety Rating Scale (HARS) was used to determine the severity of associated anxiety symptoms of the patient and according to the scale used, all the selected patients had a moderate to severe anxiety level. Half of the patients were prescribed a dose of $1 \mathrm{mg} /$ day of Risperidone, which is a fairly low dosage considering that Risperidone can be given up to $8 \mathrm{mg}$ according to the FDA dosage limits, and the other half of given $3 \mathrm{mg} /$ day of clonazepam. Both drugs were used in combination with $50 \mathrm{mg} /$ day dosage of Sertraline which was given to all the patients in the group The Patients were followed up after 10 days of and their anxiety levels were re-evaluated on the Hamilton Anxiety Rating Scale. Moreover, the Side Effect profiling of Risperidone was also done in all the Patients by using the Glasgow Anti-psychotic Side-effect Scale (GASS)'.

\section{RESULTS}

The first group receiving sertraline and risperidone showed a significant decrease in the level of anxiety as measured by HARS with a mean score of 29.16 on day 0 to a mean score of 9.60 on day 10 . The second group receiving sertraline and clonazepam also showed a significant decrease in the anxiety levels with a mean HARS score of 31.12 on day 0 to 12.36 on day 10 .

The side effect profiling of 30 patients receiving risperidone was done using GASS. Two patients experienced mild side 
effect with a GASS score of 5 and 8 respectively.

\section{CONCLUSION/DISCUSSION}

The above mentioned results of the study provide evidence that the use of a dosage, as low as $1 \mathrm{mg}$ of Risperidone, not only showed significant decrease in the level of anxiety experienced by patients having MDD, OCD and GAD but also caused none or minimal/mild side effects in terms of EPS, drowsiness and weight-gain. These findings suggest that Low-dose administration of Risperidone in treatment of moderate to severe anxiety states associated with MDD, GAD and OCD can be considered over the use of high dose of benzodiazepines which have a high addictive potential among other side effects. Moreover, the reduction in the anxiety level of patients, as calibrated on the $10^{\text {th }}$ day, has to be attributed predominately to Risperidone or Clonazepam, as the SSRI shows its full effect only after two to three weeks.

An additional benefit in the use of low dose risperidone is that, risperidone will also augment the action of any anti-depressant that the physician may be using as a primary drug for the treatment of the Major Depressive Disorder, Obsessive Compulsive Disorder and Generalized Anxiety Disorder and render the treatment with an anti-depressant for these disorder a "push" or "head-start", so to speak.

Anxiety levels in patients with OCD showed maximum amelioration with risperidone. This could be attributed to a possible anti-obsessional effect of risperidone. There is some evidence that adding quetiapine or risperidone to antidepressants increases its efficacy, but this must be weighed against less tolerability and limited data [8].

The unprecedented and unaccounted use of benzodiazepines is running havoc in many countries in terms of substance abuse problems and is exponentially increasing as more and more people are getting introduced to this class of drugs. The prescription of benzodiazepine should be minimized as much as possible to counter the related addiction problems and the search for and use of better alternatives should be incorporated into clinical practice of Psychiatry.

The challenge in this suggested usage of risperidone remains the social stigma attached with the usage of antipsychotics, personal biases of treating physicians and lack of scientific documentations in favor of this algorithm at a larger scale.

\section{CONFLICT OF INTEREST}

Declared None.

\section{ACKNOWLEDGEMENTS}

Declared None.

\section{REFERENCES}

[1] Sadock BJ, Sadock VA, Ruiz P, Eds. Kaplan \& Saddock Comprehensive Textbook of Psychiatry. 9th ed. USA: Amazon 2009.

[2] Diagnostic and Statistical Manual of Mental Disorders. Washington, D.C.: American Psychiatric Association 2013.

[3] Lader M. Fortnightly review: Treatment of anxiety. BMJ 1994; 309(6950): 321-4. DOI: 10.1136/bmj.309.6950.321

[4] Breier A. Anxiety disorders and antipsychotic drugs: A pressing need for more research. Am J Psychiatry 2011; 168(10): 1012-4. DOI: 10.1176/appi.ajp.2011.11071049

[5] Comer JS, Mojtabai R, Olfson M. National trends in the antipsychotic treatment of psychiatric outpatients with anxiety disorders. Am J Psychiatry 2011: 168(10): 1057-65.

DOI: 10.1176/appi.ajp.2011.11010087

[6] The American Psychiatric Association Practice Guidelines for the Psychiatric Evaluation of Adults. $3^{\text {rd }}$ ed. USA: American Psychiatric Association 2015.

DOI: 10.1176/appi.books.9780890426760.pe02

[7] Stahl SM. Stahl's essential psychopharmacology: Neuroscientific basis and practical applications. UK: Cambridge University Press 2013

[8] Komossa K, Depping AM, Meyer M, Kissling W, Leucht S. Second-generation antipsychotics for obsessive compulsive disorder. Cochrane Database Syst Rev 2010; (2): CD008141. DOI: 10.1002/14651858.CD008141.pub2 